\title{
A Modified Barrier and Barrier Method and the Optimal Power Flow Problem
}

\author{
J. A. Delgado, E. C. Baptista, G. F. Bregadioli, L. Nepomuceno, Member, IEEE, A. R. Balbo and \\ E. M. Soler
}

\begin{abstract}
This work proposes an approach that uses a union of methods based on logarithmic barrier function and modified logarithmic barrier function for the resolution of the reactive optimal power flow problem. In this proposed approach the inequality constraints are transformed into equalities by adding the slack variables, which are handled by logarithmic barrier function or modified logarithmic barrier function, and the equality constraints are handled by means of Lagrange multipliers. These methods are used in two steps. Initially, the method based on the logarithmic barrier function is applied until the transition condition is satisfied. After the method based on modified logarithmic barrier function is used until the stop condition is satisfied. Numerical tests carried out with the IEEE $14,30,57$ and 118 bus electrical systems indicate that the proposed approach is efficient in the resolution of the reactive optimal power flow problem and the results obtained by the proposed approach were compared with the logarithmic barrier method and the logarithmic barrier method modified, separately.
\end{abstract}

Keywords - logarithmic barrier method, modified logarithmic barrier method, Reactive Optimal Power Flow, Electrical Power System.

\section{INTRODUÇÃO}

$\mathrm{O}$ FLUXO de Potência Ótimo (FPO) pode ser representado matematicamente como um problema de otimização não linear, restrito, não convexo e de grande porte, com variáveis contínuas ou discretas. Ele otimiza uma determinada função objetivo, a qual satisfaz algum desempenho do sistema, e satisfaz as restrições de igualdade - equações de fluxo de potência - e as de desigualdade restrições físicas e operacionais do sistema. Segundo [1] diferentes classes de problemas de FPO, para diferentes aplicações, podem ser determinadas ao selecionar diferentes funções a serem minimizadas, e diferentes conjuntos de controles e restrições.

Neste trabalho exploramos um caso particular do FPO o qual é denominado de FPO Reativo (FPOR). Neste, os controles ativos são fixados e as variáveis de controle relacionadas com a potência reativa como: as magnitudes da tensão dos geradores e dos compensadores síncronos e estáticos de potência reativa e os taps dos transformadores; são otimizados em relação à uma função objetivo. Neste trabalho será utilizada como função objetivo a função de perdas de potência ativa na transmissão.

O problema de FPO teve sua primeira formulação no início da década de 60 por [2], a partir da incorporação das equações

J. A. Delgado, Universidade Estadual Paulista(Unesp), Bauru, SP, Brasil, jessica_dellgado@hotmail.com;

E. C. Baptista, Universidade Estadual Paulista(Unesp), Bauru, SP, Brasil, baptista@fc.unesp.br;

G. F. Bregadioli, Universidade Estadual Paulista(Unesp), Bauru, SP, Brasil, bregadioli.gabriela@gmail.com ,

L. Nepumuceno, Universidade Estadual Paulista(Unesp), Bauru, SP, de fluxo de potência ao problema de despacho econômico. Carpentier resolveu este problema ao utilizar a função Lagrangiana, as condições de Karush-Khun-Tucher (KKT) e o método de Gauss-Seidel. A partir deste, vários trabalhos, os quais utilizam diferentes abordagens de otimização na resolução do FPO, foram desenvolvidos. Uma das primeiras abordagens propostas para resolução do FPO utilizou o método do gradiente reduzido e é apresentada em [3]. O autor de [4] utilizou o método de penalidade e foi o primeiro a trabalhar com a matriz Hessiana da função Lagrangiana. A utilização do método de Newton com as condições de KKT pode ser encontrado no trabalho de [5], uma das vantagens do algoritmo é a utilização da técnica de esparsidade na matriz Hessiana, porém a fragilidade é que em todas as iterações necessita identificar as restrições de desigualdade ativas na solução. A função Lagrangiana Aumentada foi explorada em [6] para resolução deste problema. $\mathrm{Na}$ década de 80 , o trabalho de [7] iniciou uma nova linha de pesquisa que ficou conhecida como Método de Pontos Interiores (MPIs). Esse tipo de método já havia sido proposto em [8] e [9], porém devido à problemas de mal condicionamento da matriz Hessiana passaram a não serem utilizados. Isso se modificou após o trabalho de Karmarkar. Segundo [10], os MPIs revolucionaram o campo da otimização. No inicio da década de 90, o autor de [11] foi o primeiro a utilizar um método da classe dos MPIs, denominado de primaldual barreira logarítmica (PDBL), na resolução do FPO. As vantagens do PDBL é a facilidade de tratar as restrições de desigualdade pela função barreira logarítmica, a sua velocidade de convergência e o fato que o ponto inicial não necessita ser estritamente interior. Porém, apresenta desvantagens tais como: a sensibilidade ao parâmetro de barreira, em alguns casos o método pode divergir e a necessidade da garantia das folgas serem não negativas em todo o processo iterativo.

Diversos autores utilizaram os MPIs associado a outras técnicas na resolução e análise da solução dos problemas de FPO com sucesso. Entre eles citamos: [12] que utilizaram o método prima-dual previsor-corretor; [13] com o método de ponto interior infactível para o FPO linearizado; [14] os quais abordaram o FPO em coordenadas retangulares e o resolveram com MPIs; [15] que utilizou o método de pontos interiores com múltiplas correções centrais; [16] que aplicou o método de ponto interior de caminho central ao problema de despacho do FPO; [17] os quais utilizaram o MPIs com região de confiança; [18] os quais propuseram a associação do método da

Brasil, leo@feb.unesp.br;

A. R Balbo, Universidade Estadual Paulista(Unesp), Bauru, SP, Brasil, arbalbo@fc.unesp.br.

E. M Soler, Universidade Estadual Paulista(Unesp), Bauru, SP, Brasil, edilaine@fc.unesp.br . 
Lagrangiana aumentada ao método PDBL pra resolver o FPO reativo; [19] que analisaram três MPIs: PDBL, primal-dual previsor-corretor e primal-dual com múltiplas correções centralizadas e compararam seus resultados; [20] que utilizaram o método do gradiente reduzido em conjunto com a Lagrangiana aumentada e o PDBL; [21] que realizou uma análise de sensibilidade na solução do FPO obtida através do método PDBL; [22] que utilizaram o método primal-dual previsor-corretor com o sistema de KKT relaxado e resolveu o FPO dinâmico; [23] que determinou uma abordagem baseada no PDBL e resolveu o FPO com incertezas; [24] que discutiu a aplicação dos MPIs com múltiplas correções centrais nos problemas de FPO e FPO com restrição de segurança; [25] que utilizou o PDBL convencional, com estratégias adaptativas de atualização do parâmetro de barreira, método de busca linear com filtro e a restauração da factibilidade na resolução do FPO, quando aplicado a sistemas flexíveis de transmissão $\mathrm{AC}$, e finalmente [26] que utilizou o método PDBL associado a uma função penalidade para tratar as variáveis discretas.

Outra classe de métodos, que surgiu com o trabalho de [27], é denominada de princípio de reescalamento não linear. Segundo [28], esta classe de métodos transforma a função objetivo e/ou as restrições de um problema de otimização em outro problema equivalente, o qual tem o mesmo conjunto de soluções ótimas. O princípio de reescalamento inclui como caso especial a classe da função barreira modificada apresentada em [27]. Essa função foi explorada na resolução do FPOR por [21] que utilizou a função barreira logarítmica modificada para tratar as restrições de desigualdade e as demais restrições tratou com multiplicadores de Lagrange, e denominou de método da função Lagrangiana barreira logarítmica modificada (FLBLM).

Outros trabalhos utilizaram a função barreira modifica para resolver de maneira eficiente o FPO como: [29] que exploraram o método da função Lagrangiana aumentada barreira modificada para resolver o problema de seleção de tap do transformador ótimo; [30] que aplicaram o algoritmo primaldual barreira logarítmica modificada previsor-corretor; [31] o qual utilizou o método da função barreira modificada associado ao método de penalidade; [32] que aplicou FLBLM e analisou o condicionamento da matriz Hessiana; [33] propôs uma abordagem que utiliza uma função barreira modificada, a qual denominou de método de ponto interior com barreira de segurança; [34] que utilizou uma função barreira modificada relaxada e resolveu o FPO DC com sobrecarga e, por fim, [35] o qual também utilizou a função barreira logarítmica modificada com procedimento previsor-corretor e acrescentou uma estratégia de convergência global para resolver o FPOR.

Assim, tendo em vista a importância do FPO e a busca por métodos mais eficientes e robustos de solução é proposto neste trabalho o método primal-dual barreira logarítmica e barreira logarítmica modificada (PDBLBLM), que consiste na união dos métodos PDBL e da FLBLM. Este método justifica-se tendo em vista que uma proposta similar já foi investigada por [36] Neste, o autor propõe a união do método PDBL com o método da função barreira modificada em um único algoritmo para resolver problemas de programação não linear. Em sua conclusão [36] destaca que a união dos métodos consegue resolver um número maior de problemas do que quando comparado aos métodos separadamente.
Além da motivação de [36], os métodos PDBL e da FLBLM já foram investigados por vários autores separadamente, e são considerados métodos eficientes, tanto na resolução de problemas de otimização não lineares, quanto do FPO. O objetivo de unir os dois métodos em um, é aproveitar as melhores qualidades de cada método e tentar acelerar a convergência do algoritmo.

No método proposto, as restrições de desigualdade do problema são transformadas em igualdade através da utilização de variáveis de folga não negativas. As variáveis de folga são tratadas pelas funções barreira logarítmica ou barreira logarítmica modificada, respectivamente, e as demais restrições através dos multiplicadores de Lagrange. Estes métodos são utilizados em duas etapas. Inicialmente aplica-se o método PDBL até que um critério de transição seja satisfeito, em seguida aplica-se o método da FLBLM até que um critério de parada seja satisfeito. Este método difere do apresentado em [36] por aplicar a função barreira logarítmica nas variáveis de folga e não nas restrições. Além disso, os critérios de parada para os métodos são distintos e, consequentemente o algoritmo resultante é diferente.

Testes numéricos com os sistemas elétricos IEEE 14, 30, 57 e 118 barras foram realizados e os resultados obtidos foram comparados com os resultados dos métodos PDBL e da FLBLM, separadamente, e comprovam a eficiência do método proposto em obter soluções de boa qualidade, com bom desempenho computacional.

Este trabalho encontra-se dividido da seguinte maneira: na seção II é apresentado o modelo matemático de FPOR; nas seções III e IV são apresentados os métodos PDBL e da FLBLM respectivamente; na seção $\mathrm{V}$ é proposto o método PDBLBLM; na seção VI é apresentado a implementação dos algoritmos; finalmente os testes e as conclusões são apresentadas nas seções VII e VIII respectivamente.

\section{O MODELO MATEMÁTICO DO FPO REATIVO}

O problema de FPOR, considerado neste trabalho, utiliza a função objetivo de perdas de potência ativa na transmissão. Segundo [37] esta função é não separável e não permite simplificações, e com esta função o FPOR pode ser considerado uma das variantes mais difíceis de ser resolvida. Em (1) é apresentado o modelo matemático para o problema de FPO:

$$
\begin{aligned}
\text { Minimizar } & f(V, \theta, \text { tap }) \\
\text { sujeito } a: & \begin{cases}\Delta P_{i}(V, \theta, \text { tap })=0 & i=1,2, \ldots, N B C C R \\
\Delta Q_{j}(V, \theta, \text { tap })=0 & j=1,2, \ldots, N B C \\
Q_{k}^{\text {min }} \leq Q_{k}(V, \theta, \text { tap }) \leq Q_{k}^{\max }, & k=1,2, \ldots, N B C R \\
V_{p}^{\min } \leq V_{p} \leq V_{p}^{\max } & p=1,2, \ldots, N B \\
\operatorname{tap}_{q}^{\min } \leq \operatorname{tap}_{q} \leq \operatorname{tap}_{q}^{\max } & q=1,2, \ldots, N T\end{cases}
\end{aligned}
$$

em que:

$N B$ é o número de barras do sistema elétrico;

$N B C$ é o número de barras de carga;

$N B C R$ é o número de barras de controle de reativo;

$N B C C R$ é o número de barras de carga e de controle de reativos; 
NT é o número de transformadores com tap variável; $Q_{k}^{\min } e Q_{k}^{\max }$ são os limites inferiores e superiores de geração de potência reativa, respectivamente;

$V_{p}^{\min } e V_{p}^{\max }$ são os limites inferiores e superiores das magnitudes da tensão, respectivamente;

$\operatorname{tap}_{p}^{\min } e \operatorname{tap}_{p}^{\max }$ são os limites inferiores e superiores dos taps dos transformadores, respectivamente.

As variáveis do problema são:

tap $=\left(\operatorname{tap}_{1}, \operatorname{tap}_{2}, \ldots, \operatorname{tap}_{N T}\right)^{t}$ é $\quad \mathrm{o} \quad$ vetor dos taps dos transformadores $1,2, \ldots, N T$;

$V=\left(V_{1}, V_{2}, \ldots, V_{N B}\right)^{t}$ é o vetor das magnitudes da tensão nas barras $1,2, \ldots, N B$;

$\theta=\left(\theta_{1}, \theta_{2}, \ldots, \theta_{N B}\right)^{t}$ é o vetor dos ângulos da tensão nas barras $1,2, \ldots, N B$.

As funções de (1) são descritas a seguir:

- Função objetivo de perdas de potência ativa na transmissão:

$$
f(V, \theta, \text { tap })=\sum_{k m \in \Omega} g_{k m}\left(\frac{1}{\operatorname{tap}^{2}} V_{k}^{2}+V_{m}^{2}-2 \frac{1}{\operatorname{tap}} V_{k} V_{m} \cos \theta_{k m}\right)
$$

- Equações de balanço de potência ativa nas barras:

$$
\begin{aligned}
& \Delta P_{i}(V, \theta, \text { tap })=P_{i}^{G}-P_{i}^{C}-\sum_{m \in \Omega_{i}} P_{i m}(V, \theta, \text { tap }), \\
& \text { com } \begin{aligned}
P_{i m}(V, \theta, \text { tap })= & \left(\operatorname{tap}_{i m} V_{i}\right)^{2} g_{\text {im }}- \\
& \left(\operatorname{tap}_{\text {im }} V_{i}\right) V_{m}\left(g_{\text {im }} \cos \theta_{i m}+b_{i m} \operatorname{sen} \theta_{i m}\right) ;
\end{aligned}
\end{aligned}
$$

- Equações de balanço de potência reativa nas barras:

$$
\begin{gathered}
\Delta Q_{j}(V, \theta, \text { tap })=Q_{j}^{G}+b_{j}^{s h} V_{j}^{2}-Q_{j}^{C}-\sum_{m \in \Omega_{j}} Q_{j m}(\operatorname{tap}, V, \theta), \\
\operatorname{com} Q_{j m}=-\left(\operatorname{tap}_{j m} V_{j}\right)^{2}\left(b_{j m}+b_{j m}^{s h}\right)+ \\
\left(\operatorname{tap}_{j m} V_{j}\right) V_{m}\left(b_{j m} \cos \theta_{j m}-g_{j m} \operatorname{sen} \theta_{j m}\right) ;
\end{gathered}
$$

- Geração de Potência reativa nas barras:

$$
\begin{gathered}
Q_{k}=(\operatorname{tap}, V, \theta)=Q_{k}^{C}-b_{k}^{s h} V_{k}^{2}+\sum_{m \in \Omega_{k}}-\left(t_{k m} V_{k}\right)^{2}\left(b_{k m}+b_{k m}^{s h}\right)+ \\
\left(\operatorname{tap}_{k m} V_{k}\right) V_{m}\left(b_{k m} \cos \theta_{k m}-g_{k m} \operatorname{sen} \theta_{k m}\right) .
\end{gathered}
$$

em que: $\Omega$ é o conjunto de todas as linhas de transmissão; $\Omega_{k}$ é o conjunto de todas as barras ligadas à barra $k ; g_{k m}, b_{k m} e b_{k m}^{s h}$ são a condutância e as susceptâncias da linha $k m ; P_{k}^{G} e P_{k}^{C}$ são as potências ativas geradas e consumidas, respectivamente; $Q_{k}^{G} e Q_{k}^{C}$ são as potências reativas geradas e consumidas, respectivamente.
Matematicamente, para definirmos os métodos de resolução, o problema (1) é representado por:

\section{Minimizar $f(x)$}

$$
\text { sujeito a : }\left\{\begin{array}{l}
g(x)=0 \\
h(x) \leq 0 \\
x^{\min } \leq x \leq x^{\max }
\end{array}\right.
$$

em que: o vetor $x \in \square^{n}$, onde $x=(V, \theta$,tap $)$ representa as variáveis de estado e de controle; $g(x)=0$, com $g(x) \in \square^{m}$, representa as restrições de igualdade; $h(x) \leq 0$, com $h(x) \in \square^{r}$, representa as restrições de desigualdade e $x^{\min }$ e $x^{\max }$ são os limites inferiores e superiores das variáveis respectivamente.

A função objetivo, $f(x)$, representa as perdas de potência ativa na transmissão. Nas próximas seções são apresentados os métodos PDBL e da FLBLM, os quais são utilizados no método PDBLBLM proposto neste trabalho para resolução do FPO reativo.

\section{O MÉTODO PRIMAL-DUAL BARREIRA LOGARÍTMICA}

No método PDBL, as restrições de desigualdade de (2) são transformadas em igualdade utilizando variáveis de folga não negativas. Com isso têm-se o seguinte problema modificado, dado em (3).

$$
\begin{aligned}
\text { Minimizar } & f(x) \\
\text { sujeito } a: & \left\{\begin{array}{l}
g(x)=0 \\
h(x)+s_{1}=0 \\
x+s_{2}=x^{\max } \\
-x+s_{3}=-x^{\min } \\
s_{1} \geq 0 \\
s_{2} \geq 0 \\
s_{3} \geq 0
\end{array}\right.
\end{aligned}
$$

sendo os vetores $s_{1} \in \square^{r}, s_{2} \in \square^{n}$ e $s_{3} \in \square^{n}$. Os vetores $s_{1}, s_{2}$ e $s_{3}$ são das variáveis de folga.

$\mathrm{Na}$ sequência as variáveis de folgas não negativas são tratadas através da função barreira logarítmica, como em (4):

$$
\begin{aligned}
& \text { Minimizar } f(x)-\mu \sum_{j=1}^{r} \ln \left(s_{1_{j}}\right)-\mu \sum_{i=1}^{n} \ln \left(s_{2_{i}}\right)-\mu \sum_{i=1}^{n} \ln \left(s_{3_{i}}\right) \\
& \text { sujeito } a:\left\{\begin{array}{l}
g(x)=0 \\
h(x)+s_{1}=0 \\
x+s_{2}=x^{\max } \\
-x+s_{3}=-x^{\min }
\end{array}\right.
\end{aligned}
$$


Associa-se ao problema (4) a seguinte função Lagrangiana barreira logarítmica (FLBL):

$$
\begin{aligned}
F L B L= & f(x)-\mu \sum_{j=1}^{r} \ln \left(s_{1_{j}}\right)-\mu \sum_{i=1}^{n} \ln \left(s_{2_{i}}\right)-\mu \sum_{i=1}^{n} \ln \left(s_{3_{i}}\right)+ \\
& \lambda^{t} g(x)+\pi_{1}^{t}\left(h(x)+s_{1}\right)+\pi_{2}^{t}\left(x+s_{2}-x_{\max }\right)+ \\
& \pi_{3}^{t}\left(-x+s_{3}+x_{\text {min }}\right)
\end{aligned}
$$

sendo: $\lambda \in \square^{m}, \pi_{1} \in \square_{+}^{r}, \pi_{2} \in \square_{+}^{n}$ e $\pi_{3} \in \square_{+}^{n}$ os vetores dos multiplicadores de Lagrange, $\mu$ o parâmetro de barreira e $s_{1}, s_{2}$ e $s_{3}$ os vetores das variáveis de folga.

A condição necessária de primeira ordem é aplicada sobre a FLBL (5), gerando o sistema de equações não lineares, dado em (6):

$\operatorname{VFLBL}\left(x, s_{1}, s_{2}, s_{3}, \lambda, \pi_{1}, \pi_{2}, \pi_{3}\right)=0$

em que:

$$
\nabla F L B L=\left[\begin{array}{c}
\nabla f(x)+J_{g}(x)^{t} \lambda+J_{h}(x)^{t} \pi_{1}+\pi_{2}+\pi_{3} \\
-\frac{\mu}{s_{1}}+\pi_{1} \\
-\frac{\mu}{s_{2}}+\pi_{2} \\
-\frac{\mu}{s_{3}}+\pi_{3} \\
g(x) \\
h(x)+s_{1} \\
x+s_{2}-x_{\max } \\
-x+s_{3}+x_{\min }
\end{array}\right]
$$

com:

$$
\begin{aligned}
& J_{g}(x)^{t}=\left(\nabla_{x} g_{1}(x), \ldots, \nabla_{x} g_{m}(x)\right) \mathrm{e} \\
& J_{h}(x)^{t}=\left(\nabla_{x} h_{1}(x), \ldots, \nabla_{x} h_{r}(x)\right),
\end{aligned}
$$

denominadas de matrizes Jacobianas.

A solução do sistema de equações não lineares é determinada através do método de Newton. O método de Newton gera as direções de busca $\left(\Delta x, \Delta s_{1}, \Delta s_{2}, \Delta s_{3}, \Delta \lambda, \Delta \pi_{1}, \Delta \pi_{2}, \Delta \pi_{3}\right)$, as quais são utilizadas para a atualização das variáveis, e resulta em um sistema matricial que, pode ser representado da seguinte forma:

$$
W \Delta d=-\nabla F L B L
$$

sendo: $\Delta d^{t}=\left(\Delta x, \Delta s_{1}, \Delta s_{2}, \Delta s_{3}, \Delta \lambda, \Delta \pi_{1}, \Delta \pi_{2}, \Delta \pi_{3}\right)$, o vetor $\nabla F L B L$ é dado por (7), e a matriz $W$ por (9):

$$
W=\left[\begin{array}{cccccccc}
\nabla_{x x}^{2} F L B L & 0 & 0 & 0 & J_{g}(x)^{t} & J_{h}(x)^{t} & I & I \\
0 & -\mu S_{1} & 0 & 0 & 0 & I & 0 & 0 \\
0 & 0 & -\mu S_{2} & 0 & 0 & 0 & I & 0 \\
0 & 0 & 0 & -\mu S_{3} & 0 & 0 & 0 & I \\
J_{g}(x) & 0 & 0 & 0 & 0 & 0 & 0 & 0 \\
J_{h}(x) & I & 0 & 0 & 0 & 0 & 0 & 0 \\
I & 0 & I & 0 & 0 & 0 & 0 & 0 \\
I & 0 & 0 & I & 0 & 0 & 0 & 0
\end{array}\right]
$$

a qual é denominada de matriz Hessiana da FLBL (5), com:

$$
\nabla_{x x}^{2} F L B L=\nabla_{x x}^{2} f(x)+\sum_{p=1}^{m} \lambda_{p} \nabla_{x x}^{2} g_{p}(x)+\sum_{j=1}^{r} \pi_{1_{j}} \nabla_{x x}^{2} h_{j}(x),
$$

e as submatrizes $S_{1}, S_{2}$ e $S_{3}$ são dadas por:

$$
\begin{aligned}
& S_{1}=\left(\begin{array}{ccc}
\frac{\mu}{\left(s_{1_{1}}\right)^{2}} & & 0 \\
& \ddots & \\
0 & & \frac{\mu}{\left(s_{1_{p}}\right)^{2}}
\end{array}\right), S_{2}=\left(\begin{array}{ccc}
\frac{\mu}{\left(s_{2_{1}}\right)^{2}} & & 0 \\
& \ddots & \\
0 & & \frac{\mu}{\left(s_{2_{r}}\right)^{2}}
\end{array}\right), \\
& S_{3}=\left(\begin{array}{ccc}
\frac{\mu}{\left(s_{3_{1}}\right)^{2}} & \\
& \ddots & \frac{\mu}{\left(s_{3_{r}}\right)^{2}}
\end{array}\right) .
\end{aligned}
$$

Os vetores das variáveis $x, s, \lambda$ e $\pi$ são atualizados por (10):

$$
\begin{aligned}
x^{k+1} & =x^{k}+\alpha_{p} \Delta x^{k} & \lambda^{k+1} & =\lambda^{k}+\alpha_{d} \Delta \lambda^{k} \\
s_{1}^{k+1} & =s_{1}^{k}+\alpha_{p} \Delta s_{I}^{k} & \pi_{1}{ }^{k+1} & =\pi_{1}^{k}+\alpha_{d} \Delta \pi_{1}^{k} \\
s_{2}^{k+1} & =s_{2}^{k}+\alpha_{p} \Delta s_{2}^{k} & \pi_{2}{ }^{k+1} & =\pi_{2}^{k}+\alpha_{d} \Delta \pi_{2}^{k} \\
s_{3}^{k+1} & =s_{3}^{k}+\alpha_{p} \Delta s_{3}^{k} & \pi_{3}{ }^{k+1} & =\pi_{3}^{k}+\alpha_{d} \Delta \pi_{3}^{k}
\end{aligned}
$$

em que: $\alpha_{p}$ e $\alpha_{d}$ são os passos utilizados na atualização das variáveis primais e duais, respectivamente.

Para a determinação dos passos primais e duais adota-se uma adaptação da estratégia utilizada por [1], [14], entre outros.

O objetivo do passo primal é garantir que as variáveis de folga sejam não negativas. Assim esse passo é dado por:

$$
\alpha_{p}=\psi \min \left\{\left(\min _{\Delta S_{1}<0} \frac{-s_{1}}{\Delta s_{1}}, \min _{\Delta S_{2}<0}<\frac{-S_{2}}{\Delta s_{2}}, \min _{\Delta S_{3}<0}<\frac{-S_{3}}{\Delta s_{3}}, 1\right\} .\right.
$$


O passo dual é calculado de forma que cada componente dos vetores duais $\pi_{1}, \pi_{2}$ e $\pi_{3}$ permaneçam com seus respectivos sinais, isto é:

$$
\alpha_{d}=\psi \min \left\{\left(\min _{\Delta \pi_{l}<0 e \pi_{l}>0} \frac{-\pi_{1}}{\Delta \pi_{1}}, \min _{\Delta \pi_{2}<0 e \pi_{l}>0} \frac{-\pi_{2}}{\Delta \pi_{2}}, \min _{\Delta \pi_{l}<0} \frac{-\pi_{3}}{\Delta \pi_{l}>0}, 1\right)\right\} .
$$

em que $\psi=0,9995$.

A atualização do parâmetro de barreira $\mu$ é dada por:

$$
\mu^{k+1}=\frac{\mu^{k}}{\beta}
$$

em que: $\beta>1$.

As condições $s_{i}>0, i=1,2,3$, devem ser satisfeitas em todas as iterações.

O critério de parada do método, conforme apresentado por [14], é da seguinte forma: $e_{1}^{k} \leq \xi_{1}, \operatorname{com} e_{1}=\left\|g\left(x^{k}\right)\right\|_{\infty}$

$e_{2}^{k} \leq \xi_{2}, \quad \operatorname{com} e_{2}=\frac{\left|f\left(x^{k}\right)-f\left(x^{k-1}\right)\right|}{1+\left|f\left(x^{k}\right)\right|} \mathrm{e}$

$e_{3}^{k} \leq \xi_{3}, \operatorname{com} e_{3}=\left\|h\left(x^{k}\right)\right\|_{\infty}$.

em que: $\xi_{1}>0, \xi_{2}>0$ e $\xi_{3}>0$ são denominados de tolerância e assumem um valor pequeno pré-estabelecido. Se os critérios (14), (15) e (16) são satisfeitos, então a factibilidade primal e dual estão garantidas, o que significa que na iteração $k$ têm-se uma solução que satisfaz as condições de KKT.

A seguir é apresentado o Algoritmo PDBL.

\section{A. Algoritmo PDBL}

Dado o problema (2), construa a FLBL (5).

Faça $k=0, \xi_{1}>0, \xi_{2}>0, \xi_{3}>0$;

Obtenha uma estimativa inicial para $d^{0}=\left(x^{0}, s_{1}^{0}, s_{2}^{0}, s_{3}^{0}, \lambda^{0}, \pi_{1}^{0}, \pi_{2}^{0}, \pi_{3}^{0}\right)$ e $\mu^{0}$.

1 Enquanto (14), (15) e (16) não são satisfeitas faça

2 Resolva o sistema (8);

3 Calcule os passos $\alpha_{p}$ e $\alpha_{d}$ utilizando (11) e (12);

$4 \quad$ Atualize as variáveis por (10);

$5 \quad$ Atualize o parâmetro de barreira por (13);

$6 \quad$ Faça $\mathrm{k}=\mathrm{k}+1$;

7 Fim

$8 x^{k}$ é solução para do problema (2).

\section{O MÉTODO DA FUNÇÃO LAGRANGIANA BARREIRA LOGARÍTMICA}

No método da FLBLM, as restrições de desigualdade de (2) são transformadas em igualdade utilizando variáveis de folga não negativas. Com isso têm-se o seguinte problema modificado, dado em (3). Na sequência, aplica-se uma relaxação, nas condições de não negatividade do problema (3), utilizando o parâmetro de barreira e obtêm-se:

$$
\begin{aligned}
& \text { Minimizar } f(x) \\
& \text { sujeito } a:\left\{\begin{array}{l}
g(x)=0 \\
h(x)+s_{I}=0 \\
x+s_{2}=x^{\max } \\
-x+s_{3}=-x^{\min } \\
s_{1} \geq-\mu \\
s_{2} \geq-\mu \\
s_{3} \geq-\mu
\end{array}\right.
\end{aligned}
$$

em que: $\mu>0$ é o parâmetro de barreira.

O método proposto por [27] é utilizado para transformar o problema (16), no seguinte problema modificado:

$$
\begin{array}{ll}
\text { Minimizar } & f(x) \\
\text { sujeito } a:\left\{\begin{array}{l}
g(x)=0 \\
h(x)+s_{1}=0 \\
x+s_{2}=x^{\max } \\
-x+s_{3}=-x^{\min } \\
\mu \ln \left(\mu^{-1} s_{1}+1\right) \geq 0 \\
\mu \ln \left(\mu^{-1} s_{2}+1\right) \geq 0 \\
\mu \ln \left(\mu^{-1} s_{3}+1\right) \geq 0
\end{array}\right.
\end{array}
$$

Associado ao problema (18) têm-se a seguinte função Lagrangiana barreira logarítmica modificada (FLBLM):

$$
\begin{aligned}
& F L B L M=f(x)-\mu \sum_{j=1}^{r} u_{1_{j}} \ln \left(\mu^{-1} s_{1_{j}}+1\right)-\mu \sum_{i=1}^{n} u_{2_{i}} \ln \left(\mu^{-1} s_{2_{i}}+1\right) \\
& -\mu \sum_{i=1}^{n} u_{3_{i}} \ln \left(\mu^{-1} s_{3_{i}}+1\right)+\lambda^{t} g(x)+\pi_{1}^{t}\left(h(x)+s_{1}\right)+ \\
& \pi_{2}^{t}\left(x+s_{2}-x^{\max }\right)+\pi_{3}^{t}\left(-x+s_{3}+x^{\min }\right)
\end{aligned}
$$

sendo: $u_{1} \in \square^{r}, u_{2}$ e $u_{3} \in \square^{n} \quad$ os vetores dos estimadores dos multiplicadores de Lagrange, $\lambda \in \square^{m}, \pi_{1} \in \square_{+}^{r}, \pi_{2}$ e $\pi_{3} \in \square_{+}^{n} \quad$ os $\quad$ vetores $\operatorname{dos}$ multiplicadores de Lagrange.

A condição necessária de primeira ordem é aplicada sobre a FLBLM (19), gerando um sistema de equações não lineares, sendo:

$$
\operatorname{VFLBLM}\left(x, s_{1}, s_{2}, s_{3}, \lambda, \pi_{1}, \pi_{2}, \pi_{3}\right)=0,
$$

em que: 


$$
\nabla F L B L M=\left[\begin{array}{c}
\nabla f(x)+J_{g}(x)^{t} \lambda+J_{h}(x)^{t} \pi_{1}+\pi_{2}+\pi_{3} \\
-\frac{u_{1}}{\mu^{-1} s_{1}+1}+\pi_{1} \\
-\frac{u_{2}}{\mu^{-1} s_{2}+1}+\pi_{2} \\
-\frac{u_{3}}{\mu^{-1} s_{3}+1}+\pi_{3} \\
g(x) \\
h(x)+s_{1} \\
x+s_{2}-x^{\max } \\
\left.-x+s_{3}+x^{\min }\right)
\end{array}\right]
$$

com:

$$
\begin{aligned}
& J_{1}(x)^{t}=\left(\nabla_{x} g_{1}(x), \ldots, \nabla_{x} g_{m}(x)\right) \mathrm{e} \\
& J_{1}(x)^{t}=\left(\nabla_{x} h_{1}(x), \ldots, \nabla_{x} h_{r}(x)\right),
\end{aligned}
$$

denominadas de matrizes Jacobianas.

A solução do sistema de equações não lineares (20) é determinada através do método de Newton. O método de Newton gera as direções de busca $\left(\Delta x, \Delta s_{1}, \Delta s_{2}, \Delta s_{3}, \Delta \lambda, \Delta \pi_{1}, \Delta \pi_{2}, \Delta \pi_{3}\right)$, as quais são utilizadas para a atualização das variáveis, e resulta em um sistema matricial que, pode ser representado da seguinte forma:

$$
W_{1} \Delta d=-\nabla F L B L M
$$

sendo: $\Delta d^{t}=\left(\Delta x, \Delta s_{1}, \Delta s_{2}, \Delta s_{3}, \Delta \lambda, \Delta \pi_{1}, \Delta \pi_{2}, \Delta \pi_{3}\right)$, o vetor $\nabla F L B L$ é dado por (21), e a matriz $\mathrm{W}_{1}$ por (23):

$$
\mathrm{W}_{1}=\left[\begin{array}{cccccccc}
\nabla_{x x}^{2} F L B L M & 0 & 0 & 0 & J_{g}(x)^{t} & J_{h}(x)^{t} & I & I \\
0 & -\mu S_{1} & 0 & 0 & 0 & I & 0 & 0 \\
0 & 0 & -\mu S_{2} & 0 & 0 & 0 & I & 0 \\
0 & 0 & 0 & -\mu S_{3} & 0 & 0 & 0 & I \\
J_{g}(x) & 0 & 0 & 0 & 0 & 0 & 0 & 0 \\
J_{h}(x) & I & 0 & 0 & 0 & 0 & 0 & 0 \\
I & 0 & I & 0 & 0 & 0 & 0 & 0 \\
I & 0 & 0 & I & 0 & 0 & 0 & 0
\end{array}\right]
$$

é denominada de matriz Hessiana da FLBLM (18), sendo dada por (23):

$$
\nabla_{x x}^{2} F L B L=\nabla_{x x}^{2} f(x)+\sum_{p=1}^{m} \lambda_{i} \nabla_{x x}^{2} g_{i}(x)+\sum_{j=1}^{r} \pi_{1_{j}} \nabla_{x x}^{2} h_{j}(x),
$$

e as submatrizes $S_{1}, S_{2}$ e $S_{3}$ são dadas por:

$$
S_{1}=\left(\begin{array}{ccc}
\frac{u_{1_{1}}}{\left(\mu^{-1} s_{1_{1}}+1\right)^{2}} & & 0 \\
& \ddots & \\
0 & & \frac{u_{1 r}}{\left(\mu^{-1} s_{1_{r}}+1\right)^{2}}
\end{array}\right),
$$$$
S_{2}=\left(\begin{array}{ccc}
\frac{u_{2_{1}}}{\left(\mu^{-1} s_{2_{1}}+1\right)^{2}} & & 0 \\
& \ddots & \\
0 & & \frac{u_{2 n}}{\left(\mu^{-1} s_{2_{n}}+1\right)^{2}}
\end{array}\right),
$$

e

$$
S_{3}=\left(\begin{array}{ccc}
\frac{u_{3_{1}}}{\left(\mu^{-1} s_{3_{1}}+1\right)^{2}} & & 0 \\
& \ddots & \\
0 & & \frac{u_{3 m}}{\left(\mu^{-1} s_{3_{m}}+1\right)^{2}}
\end{array}\right) .
$$

A atualização dos vetores das variáveis $x, s, \lambda$ e $\pi$ é dada por (10), como no método PDBL.

O parâmetro de barreira $\mu$ é atualizado, segundo [38] por:

$$
\mu^{k+1}=\mu^{k}\left(1-\frac{\sigma}{\sqrt{r}}\right)
$$

em que $r$ é o número de restrições de desigualdade do problema e:

$$
\sigma=\max \left(\frac{1}{\mu^{-1} s_{1_{j}}+1}\right), j=1, \ldots, r, \operatorname{para} s_{1_{j}}>0
$$

$\mathrm{O}$ vetor dos estimadores dos multiplicadores de Lagrange $u$ é atualizado pela regra de [27].

$$
u_{1 j}^{k+1}=\frac{u_{1_{j}}^{k} \mu^{k+1}}{{s_{1 j}{ }^{k+1}+\mu^{k+1}}^{k+1}}
$$

Os passos primais e duais são calculados como no método PDBL, isto é, como definido em (11) e (12).

A seguir apresenta-se o Algoritmo da FLBLM.

\section{A. Algoritmo da FLBLM}

Dado o problema (2), construa a FLBLM (19).

Faça $\mathrm{k}=0, \xi_{1}>0, \xi_{2}>0, \xi_{3}>0$; 


Obtenha uma estimativa inicial para:
\begin{tabular}{c|l}
$d^{0}=\left(x^{0}, s_{1}^{0}, s_{2}^{0}, s_{3}^{0}, \lambda^{0}, \pi_{1}^{0}, \pi_{2}^{0}, \pi_{3}^{0}\right), \mu^{0}$ e $u_{1}^{0}, u_{2}^{0}$ e $u_{3}^{0}$. \\
1 & Enquanto (14), (15) e (16) não são satisfeitas faça \\
2 & Obtenha o sistema (22) e resolva-o; \\
3 & Calcule os passos $\alpha_{p}$ e $\alpha_{d}$ utilizando (11) e (12); \\
4 & Atualize as variáveis por (10); \\
5 & Atualize o parâmetro de barreira por (24); \\
6 & Atualize os estimadores dos multiplicadores de \\
7 & Lagrange por (25); \\
8 & Faça k=k+1; \\
9 & $x_{k}$ é solução do problema (2);
\end{tabular}

\section{O MÉTODO PRIMAL DUAL BARREIRA LOGARÍTMICA E BARREIRA LOGARÍTMICA MODIFICADA}

A proposta desse artigo é unir os métodos PDBL e o da FLBLM em uma única e nova abordagem denominada de PDBLBLM. Propõe-se utilizar o método PDBL e o método da FLBLM de maneira mista. A ideia consiste em: aplicar o método de PDBL até que um critério de transição seja satisfeito e em seguida aplicar o método da FLBLM até o critério de parada (14)-(16) seja satisfeito. Para a transição do método PDBL para o da FLBLM utiliza-se o critério de que, quando (15) atingir $10^{-3}$, realiza-se a troca de métodos.

A seguir apresenta-se o Algoritmo PDBLBLM.

\section{A. Algoritmo PDBLBLM}

Dado o problema (2), construa a FLBL (5), construa a FLBLM (19)

Faça $\mathrm{k}=0, \xi_{1}>0, \xi_{2}>0, \xi_{3}>0$;

Obtenha uma estimativa inicial para: $d^{0}=\left(x^{0}, s_{1}^{0}, s_{2}^{0}, s_{3}^{0}, \lambda^{0}, \pi_{1}^{0}, \pi_{2}^{0}, \pi_{3}^{0}\right)$ e $\mu^{0}$ ；

\footnotetext{
1 Enquanto $v_{2}^{k}>\xi_{2}$ faça

2 Obtenha o sistema (8) e resolva-o;

3 Calcule os passos $\alpha_{p}$ e $\alpha_{d}$ utilizando (11) e (12);

$4 \quad$ Atualize as variáveis por (10);

5 Atualize o parâmetro de barreira por (13);

6 faça $\mathrm{k}=\mathrm{k}+1$;

7 Fim

8

9 Enquanto (14), (15) e (16) não são satisfeitas faça

10 Obtenha o sistema (22) e resolva-o;

11 Calcule os passos $\alpha_{p}$ e $\alpha_{d}$ utilizando (11) e (12);

12 Atualize as variáveis por (10);

13 Atualize o parâmetro de barreira por (24);

14 Atualize os multiplicadores de Lagrange por (25);

15 faça $\mathrm{k}=\mathrm{k}+1$;

Fim; $x^{k}$ é uma solução para o problema (2).
}

\section{IMPLEMENTAÇÃO}

Os métodos PDBL, da FLBLM e PDBLBLM foram implementados em Matlab R.2010b de acordo com os algoritmos apresentados nas seções III, IV e V, respectivamente. Todos os testes foram realizados em um computador Intel Core i5-2GHz, com 4 Gbytes de memória RAM.

$\mathrm{Na}$ implementação, para a resolução dos sistemas não lineares (6) e (20) foi utilizado o método de Newton com controle de passos dados por (11) e (12), conforme seções III e IV.

Para a montagem dos sistemas lineares, isto é, (8) e (22) foi desenvolvido um programa em Matlab que os gera a partir do banco de dados dos sistemas elétricos, disponíveis em [39]. Como as matrizes $\mathrm{W}_{\text {e }} \mathrm{W}_{1}$, apresentadas em (9) e (23) são altamente esparsas utilizou-se uma técnica de esparsidade, a qual foi introduzida por [40] e encontra-se implementada no Matlab.

Para a resolução dos sistemas (8) e (22) utilizou-se um algoritmo disponível no Matlab que realiza um tratamento especial para matrizes esparsas e utiliza diferentes métodos de solução de acordo com a estrutura da matriz dos coeficientes [41]. Desta forma, implementou-se os 3 métodos utilizando as mesmas técnicas de programação.

\section{TESTES E RESULTADOS}

A abordagem proposta foi aplicada ao problema de FPOR associado aos sistemas IEEE (Institute of Eletrical and Eletronics Engineers) 14, 30, 57 e 118 barras [39]. Para verificar a eficiência do método proposto, o PDBLBLM, os resultados obtidos foram comparados aos resultados obtidos com os métodos PDBL e da FLBLM, separadamente.

\section{A. Dados dos testes}

Para resolução de todos os testes, inicia-se o vetor das magnitudes da tensão $V_{p}=1, p=1, \ldots, N B$ os taps dos transformadores $\operatorname{tap}_{q}=1, q=1, \ldots, N T$, e os ângulos das tensões $\theta_{l}=0, \quad l=1, \ldots, N B-1$ ( em radianos). Já o parâmetro de barreira $\mu$ foi inicializado por $0,0001,0,001,0,12$ e 0,001 para os sistemas elétricos $14,30,57$ e 118 barras, respectivamente.

$\mathrm{O}$ vetor das magnitudes da tensão foram canalizados entre 0,95 pu e 1,10 pu para os sistemas IEEE 14, 30 e 57 barras, já para o IEEE 118 barras as magnitudes da tensão foram canalizadas entre $0,90 \mathrm{pu}$ e $1,10 \mathrm{pu}$. O vetor dos taps dos transformadores também foram canalizados entre $0,90 \mathrm{pu}$ e 1,05 pu. Os vetores dos multiplicadores de Lagrange foram inicializados por: $\pi_{b}=1, b=1, \ldots, M, \quad$ sendo $\mathrm{M}=$ $2 \mathrm{NB}+2 \mathrm{NT}+2 \mathrm{NBCR}$, já o vetor $\lambda_{r}=0, \quad r=1, \ldots, C$, sendo $\mathrm{C}=\mathrm{NBCCR}+\mathrm{NBC}$. $\mathrm{O}$ vetor dos estimadores dos multiplicadores de Lagrange $u_{b}=1, \quad b=1, \ldots, M$.

As variáveis de folga $s_{b}, b=1, \ldots, M$, foram determinadas através das equações: $s_{b}=y-y^{\min }$ ou $s_{b}=y+y^{\max }$, em que $y$ pode ser o valor de uma magnitude da tensão, tap ou geração 
de reativo e $y^{\min }$ ou $y^{\max }$ são os limites inferiores e superiores dessas variáveis ou restrições, respectivamente. Quando algum componente da variável de folga $s_{b}$ inicial tem o valor negativo adota-se o procedimento de aproximar pra um valor pequeno e positivo, pois o método PDBL exige que as variáveis de folga sejam não negativa, caso contrário o método não converge. $\mathrm{O}$ método da FLBLM não exige que as variáveis de folga sejam não negativas, mas $\operatorname{sim} s_{b}>-\mu$, porém para uma melhor comparação iniciou-se essa variável, nos três métodos com os mesmos valores.

$\mathrm{Na}$ Tabela I apresentam-se algumas características dos sistemas elétricos investigados. Temos que: QR são as quantidades de barras de geração de potência reativa, QT são as quantidades de taps, $\mathrm{n}$ é a quantidade total das variáveis primais e duais do método PDBL, $\mathrm{n}_{1}$ é a quantidade total das variáveis primais e duais do método da FLBLM e rdi e rdd são as quantidades de restrições de igualdade e desigualdade, respectivamente.

TABELA I

RESUMO DOS SISTEMAS ELÉTRICOS

\begin{tabular}{|c|c|c|c|c|c|c|c|c|}
\hline Sist. & Barras & Linhas & QR & QT & n & $\mathrm{n}_{1}$ & rdi & Rdd \\
\hline $\begin{array}{c}14 \\
\text { barras }\end{array}$ & 14 & 17 & 4 & 3 & 140 & 185 & 22 & 44 \\
\hline $\begin{array}{c}30 \\
\text { barras }\end{array}$ & 30 & 37 & 5 & 4 & 272 & 350 & 53 & 100 \\
\hline $\begin{array}{c}57 \\
\text { barras }\end{array}$ & 57 & 80 & 6 & 17 & 556 & 716 & 106 & 162 \\
\hline $\begin{array}{c}118 \\
\text { barras }\end{array}$ & 118 & 186 & 53 & 9 & 1150 & 1512 & 181 & 362 \\
\hline
\end{tabular}

\section{B. Resultados numéricos}

$\mathrm{Na}$ Tabela II são apresentados os resultados dos problemas de FPO associados aos sistemas elétricos IEEE de 14, 30, 57 e 118 barras, em relação ao número de iterações (It.), perdas e tempo computacional $\left(\mathrm{t}_{\mathrm{cpu}}\right)$ em segundos, para os métodos PDBL, da FLBLM e PDBLBLM.

TABELA II

PROCESSO DE OTIMIZAÇÃO

\begin{tabular}{|c|c|c|c|c|c|c|c|c|c|}
\hline \multirow{2}{*}{$\begin{array}{c}\text { Sist. } \\
\text { (barras) }\end{array}$} & \multicolumn{3}{|c|}{ PDBL } & \multicolumn{4}{c|}{ FLBLM } & \multicolumn{3}{c|}{ PDBLBLM } \\
\cline { 2 - 10 } & It. & $\begin{array}{c}\text { Perdas } \\
\text { MW }\end{array}$ & $\mathrm{t}_{\mathrm{cpu}}(\mathrm{s})$ & It. & $\begin{array}{c}\text { Perdas } \\
\text { MW }\end{array}$ & $\mathrm{t}_{\mathrm{cpu}}(\mathrm{s})$ & It. & $\begin{array}{c}\text { Perdas } \\
\text { MW }\end{array}$ & $\mathrm{t}_{\mathrm{cpu}}(\mathrm{s})$ \\
\hline 14 & 12 & 12,29 & 0,15 & 10 & 12,30 & 0,16 & 10 & 12,29 & 0,14 \\
\hline 30 & 13 & 16,13 & 0,34 & 13 & 16,15 & 0,34 & 11 & 16,13 & 0,31 \\
\hline 57 & 16 & 22,83 & 1,11 & 15 & 23,30 & 0,97 & 15 & 22,83 & 0,96 \\
\hline 118 & 17 & 108,85 & 3,56 & 17 & 110,20 & 3,56 & 16 & 108,90 & 4,12 \\
\hline
\end{tabular}

Conforme pode-se observar na Tabela II, que o método proposto, PDBLBLM, foi capaz de obter uma solução em menor tempo computacional, quando comparado aos métodos PDBL e da FLBLM, exceto para o caso de 118 barras, contudo quando comparado ao número de iterações, o método proposto convergiu em menor, ou igual número de iterações.

Em termos de perdas de potência ativa, pode-se observar que o método proposto foi capaz de obter uma solução com perdas inferior, ou igual às perdas obtidas pelos métodos PDBL e da FLBLM, exceto para o caso de 118 barras. Porém, pode-se observar que a diferença entre as perdas obtidas pelo método proposto e pelo método PDBL, é inferior a $0,05 \%$, o que evidência a qualidade da solução obtida pelo método proposto.

Para ilustrar a convergência dos três algoritmos, para o Sistema Elétrico IEEE de 118 barras apresenta-se na Fig.1 o comportamento da função objetivo.

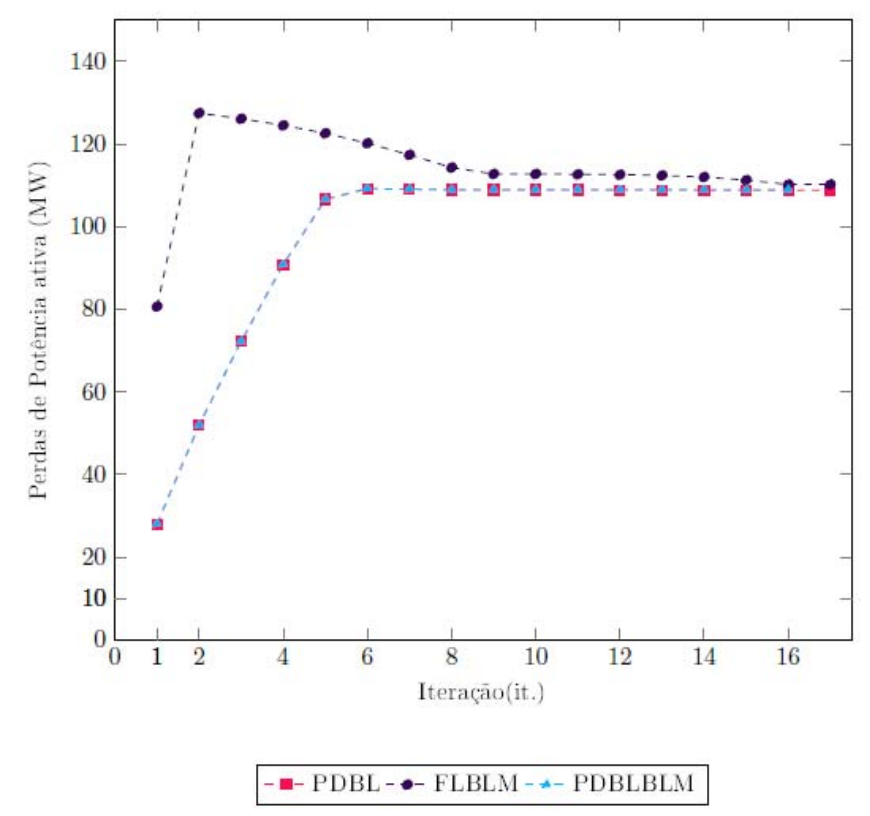

Figura 1. Convergência da função objetivo do problema de FPO associado ao Sistema Elétrico IEEE de 118 barras.

Observa-se que para todos os testes realizados as magnitudes de tensão e os taps dos transformadores obtidos, obedeceram aos seus limites. Na Fig.2, apresentam-se os resultados dessas variáveis na solução ótima para o problema de FPOR associado ao sistema elétrico IEEE de 118 barras através do método PDBLBLM.

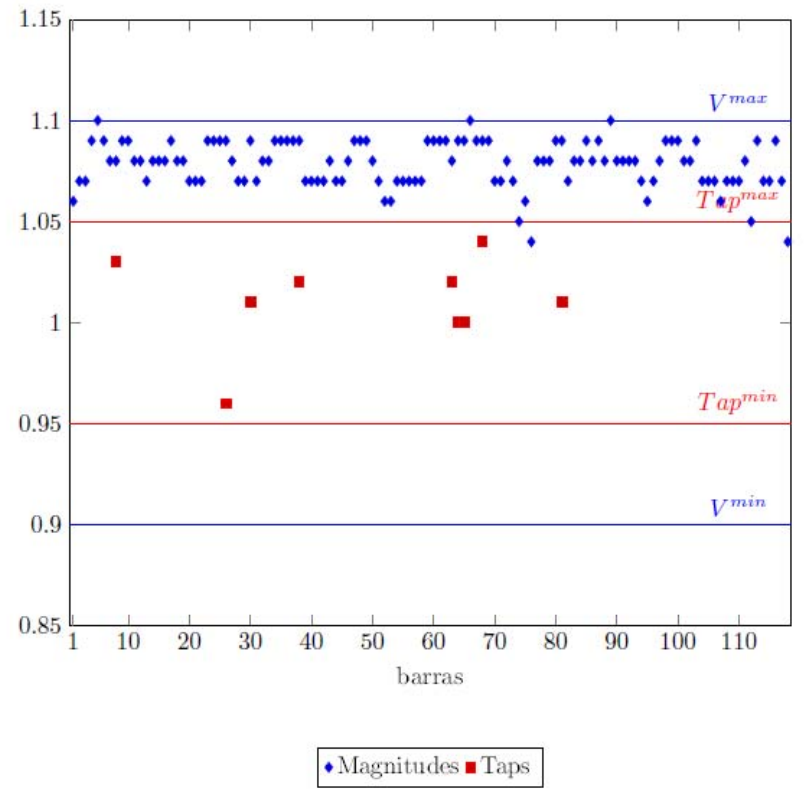

Figura 2. Magnitudes da tensão e taps do problema de FPOR associado ao sistema elétrico IEEE de 118 barras, na solução ótima. 
Com base nos resultados apresentados conclui-se que o método PDBLBLM teve um melhor resultado que os métodos PDBL e da FLBLM (quando executados em separado), quanto ao número de iterações e em relação ao tempo computacional. Assim, pode-se afirmar que o método é eficiente para a resolução do problema de FPOR.

\section{Comparação dos resultados}

O problema de FPOR, já foi resolvido na literatura através de vários métodos de otimização. Alguns destes métodos foram selecionados, para a comparação dos resultados obtidos pelo método PDBLBLM. Desta forma na Tabela III tem-se em [42] um algoritmo Genético Adaptativo Linear; em [18] um método hibrido de barreira logarítmica e Lagrangiana Aumentada; em [19] uma comparação de três métodos de pontos interiores: puro, previsor-corretor e múltiplas correções centralizadas; em [31] o método de penalidade/ barreira modificada; em [35] o método previsor-corretor barreira modificada com estratégia de convergência global; já em [26] o método primal-dual barreira logarítmica considerando as variáveis de controle como contínuas e discretas e finalmente em [43] os métodos previsorcorretor barreira logarítmica modificada e barreira logarítmica.

TABELA III

COMPARAÇÃO DO PDBLBLM COM TRABALHOS DA LITERATURA

\begin{tabular}{|c|c|c|c|c|c|c|c|c|}
\hline Sist. & $\begin{array}{c}\text { PDBL } \\
\text { BLM }\end{array}$ & $\begin{array}{c}\text { Ref. } \\
{[42]}\end{array}$ & $\begin{array}{c}\text { Ref. } \\
{[18]}\end{array}$ & $\begin{array}{c}\text { Ref. } \\
{[19]}\end{array}$ & $\begin{array}{c}\text { Ref. } \\
{[31]}\end{array}$ & $\begin{array}{c}\text { Ref. } \\
{[35]}\end{array}$ & $\begin{array}{c}\text { Ref. } \\
{[26]}\end{array}$ & $\begin{array}{c}\text { Ref. } \\
{[43]}\end{array}$ \\
\hline 14 & 12,29 & $\begin{array}{c}13,2 \\
1\end{array}$ & - & - & 12,68 & 12,29 & 12,27 & - \\
\hline 30 & 16,13 & - & 17,57 & - & 16,65 & 16,08 & 16,07 & 16,65 \\
\hline 57 & 22,83 & - & - & - & - & 22,97 & - & - \\
\hline 118 & 108,9 & - & - & 116,5 & 110,00 & 107,70 & 110,6 & 110,0 \\
\hline
\end{tabular}

Na Tabela III verifica-se que o método proposto obtém as perdas de potência ativa com valores próximos ou inferiores. Somente para a referência [35] no sistema 118 barras uma perda de potência ativa maior é determinada, porém esses autores utilizaram o método previsor-corretor barreira modificada e uma estratégia de convergência global.

Observa-se ainda que, as diferenças nos valores das perdas de potência ativa da tabela III podem estar associadas à consideração de variáveis discretas ou não, limites das variáveis ou outros parâmetros do modelo.

\section{CONCLUSÃO}

Neste trabalho apresentou-se uma nova abordagem denominada de método primal-dual barreira logarítmica e barreira logarítmica modificada (PDBLBLM) para a resolução do problema de Fluxo de Potência Ótimo Reativo. Nesta abordagem as restrições de desigualdade são tratadas pela função barreira logarítmica ou pela função barreira logarítmica modificada e as restrições de igualdade são tratadas pelos multiplicadores de Lagrange.

Cabe destacar que, da mesma forma que os métodos PDBL e da FLBLM, o método PDBLBLM é sensível à inicialização e atualização do parâmetro de barreira.
Os resultados computacionais obtidos pelo método PDBLBLM para os sistemas elétricos IEEE de 14, 30, 57 e 118 barras mostram o potencial e eficiência da abordagem proposta.

\section{AGRADECIMENTOS}

Os autores agradecem a Coordenação de Aperfeiçoamento de Pessoal de Nível Superior (CAPES), ao Conselho Nacional de Desenvolvimento Científico e Tecnológico (CNPq) - (Proc. No 448645/2014-9 e 309588/2013-8 (Bolsista CNPq)) - e a Fundação de Amparo à Pesquisa do Estado de São Paulo (FAPESP) - (Proc. No 2013/18036-1 e 2014/20853-0), pelo auxílio financeiro concedido.

\section{REFERÊNCIAS}

[1] M. El-Hawary, "Optimal Power Flow", in Gregsby, L., vol. 20, New York: CRC Press, 2007, p. 1-14.

[2] J. L. Carpentier, "Contribution a l'etude du dispatching economique", Bull Soc Francaise Electr., vol. 8, p. 431-447, 1962.

[3] H. W. Dommel e W. F. Tinney, "Optimal Power Flow Solutions", IEEE Trans. Power Appar. Syst., vol. PAS-87, n 10, p. 1866-1876, out. 1968.

[4] A. M. Sasson, F. Viloria, e F. Aboytes, "Optimal Load Flow Solution Using the Hessian Matrix", IEEE Trans. Power Appar. Syst., vol. PAS92, no 1, p. 31-41, jan. 1973.

[5] D. I. Sun, B. Ashley, B. Brewer, A. Hughes, e W. F. Tinney, "Optimal Power Flow By Newton Approach", IEEE Trans. Power Appar. Syst., vol. PAS-103, nº 10, p. 2864-2880, out. 1984.

[6] A. Santos, S. Deckmann, e S. Soares, "A dual augmented Lagrangian approach for optimal power flow", IEEE Trans. Power Syst., vol. 3, n 3, p. $1020-1025$, ago. 1988.

[7] N. Karmarkar, "A New Polynomial-time Algorithm for Linear Programming", Proc. Sixt. Annu. ACM Symp. Theory Comput., p. 302 $311,1984$.

[8] K. R. Frisch, The Logarithmic Potential Method of Convex Programming. University Institute of Economics (manuscript): Oslo, Norway, 1955.

[9] A. V. Fiacco e G. P. Mccormick, Nonlinear Programming - Sequential Unconstrained Minimization Techniques. New York: Wiley, 1968.

[10] J. Gondzio, "Interior point methods 25 years later", Eur. J. Oper. Res., vol. $218, \mathrm{n}^{\circ} 3$, p. 587-601, maio 2012.

[11] S. Granville, "Optimal reactive dispatch through interior point methods", IEEE Trans. Power Syst., vol. 9, n 1, p. 136-146, fev. 1994.

[12] Y.-C. Wu, A. S. Debs, e R. E. Marsten, "A direct nonlinear predictorcorrector primal-dual interior point algorithm for optimal power flows", IEEE Trans. Power Syst., vol. 9, n 2, p. 876-883, maio 1994.

[13] X. Yan e V. H. Quintana, "An infeasible interior-point algorithm for optimal power-flow problems", Electr. Power Syst. Res., vol. 39, n 1, p. 39-46, out. 1996.

[14] G. L. Torres e V. H. Quintana, "Optimal Power Flow in Rectangular Form via an Interior Point Method", IEEE Power Syst., vol. 13, n 4, p. 1211-1218, 1998.

[15] G. L. Torres e V. H. Quintana, "On a nonlinear multiple-centralitycorrections interior-point method for optimal power flow", IEEE Trans. Power Syst., vol. 16, n 2, p. 222-228, maio 2001.

[16] R. A. Jabr, "A Primal-Dual Interior-Point Method to Solve the Optimal Power Flow Dispatching Problem", Optim. Eng., vol. 4, n 4, p. 309-336, dez. 2003.

[17] W. Min e L. Shengsong, "A trust region interior point algorithm for optimal power flow problems", Int. J. Electr. Power Energy Syst., vol. 27, no 4, p. 293-300, maio 2005.

[18] E. C. Baptista, E. A. Belati, e G. R. M. da Costa, "Logarithmic barrieraugmented Lagrangian function to the optimal power flow problem", Int. J. Electr. Power Energy Syst., vol. 27, nº 7, p. 528-532, set. 2005.

[19] F. Capitanescu, M. Glavic, D. Ernst, e L. Wehenkel, "Interior-point based algorithms for the solution of optimal power flow problems", Electr. Power Syst. Res., vol. 77, no 5-6, p. 508-517, abr. 2007.

[20] E. P. de Carvalho, A. dos Santos Júnior, e T. F. Ma, "Reduced gradient method combined with augmented Lagrangian and barrier for the optimal power flow problem", Appl. Math. Comput., vol. 200, n 2, p. 529-536, jul. 2008 . 
[21] V. A. de Sousa, E. C. Baptista, e G. R. M. da Costa, "Fluxo de potência ótimo reativo via método da função lagrangiana barreira modificada", Sba Controle Amp Autom. Soc. Bras. Autom., vol. 19, nº 1, p. 83-92, mar. 2008.

[22] C. Y. Chung, W. Yan, e F. Liu, "Decomposed Predictor-Corrector Interior Point Method for Dynamic Optimal Power Flow", IEEE Trans. Power Syst., vol. 26, n 3, p. 1030-1039, ago. 2011.

[23] A. Mohapatra, P. R. Bijwe, e B. K. Panigrahi, "Optimal Power Flow with Multiple Data Uncertainties”, Electr. Power Syst. Res., vol. 95, p. 160 167, fev. 2013.

[24] F. Capitanescu e L. Wehenkel, "Experiments with the interior-point method for solving large scale Optimal Power Flow problems", Electr. Power Syst. Res., vol. 95, p. 276-283, fev. 2013.

[25] C. Duan, W. Fang, L. Jiang, e J. Liu, “Adaptive barrier filter-line-search interior point method for optimal power flow with FACTS devices", IET Gener. Transm. Amp Distrib., vol. 9, nº 16, p. 2792-2798, dez. 2015.

[26] E. M. Soler, E. N. Asada, e G. R. M. da Costa, "Penalty-Based Nonlinear Solver for Optimal Reactive Power Dispatch With Discrete Controls", IEEE Trans. Power Syst., vol. 28, n 3, p. 2174-2182, ago. 2013.

[27] R. Polyak, "Modified barrier functions (theory and methods)", Math. Program., vol. 54, $\mathrm{n}^{\circ}$ 1-3, p. 177-222, fev. 1992.

[28] R. Polyak e M. Teboulle, "Nonlinear rescaling and proximal-like methods in convex optimization", Math. Program., vol. 76, n 2, p. 265 284, fev. 1997.

[29] M. M. Adibi, R. A. Polyak, I. A. Griva, L. Mili, e S. Ammari, “Optimal transformer tap selection using modified barrier-augmented Lagrangian method", IEEE Trans. Power Syst., vol. 18, n 1, p. 251-257, fev. 2003.

[30] A. M. de Souza, V. A. de Sousa, e G. R. M. da Costa, "Studies of cases in power systems by Sensitivity Analysis oriented by OPF", Int. J. Electr. Power Energy Syst., vol. 32, nº 9, p. 969-974, nov. 2010.

[31] G. G. Lage, V. A. de Sousa, e G. R. M. da Costa, "Optimal power flow solution using the penalty/modified barrier method", in 2009 IEEE Bucharest PowerTech, 2009, p. 1-6.

[32] V. A. de Sousa, E. C. Baptista, e G. R. M. da Costa, "Optimal reactive power flow via the modified barrier Lagrangian function approach", Electr. Power Syst. Res., vol. 84, nº 1, p. 159-164, mar. 2012.

[33] E. J. Oliveira, L. W. Oliveira, J. L. R. Pereira, L. M. Honório, I. C. Silva Junior, e A. L. M. Marcato, "An optimal power flow based on safety barrier interior point method”, Int. J. Electr. Power Energy Syst., vol. 64 , p. 977-985, jan. 2015.

[34] M. V. Coelho, A. dos S. Junior, e A. R. L. de Oliveira, "Relaxation by Modified Logarithmic Barrier Applied to the Problem of Optimal Power Flow DC with Overload", IEEE Lat. Am. Trans., vol. 13, n 3, p. 673 678, mar. 2015.

[35] R. B. N. Pinheiro, A. R. Balbo, E. C. Baptista, e L. Nepomuceno, "Interior-exterior point method with global convergence strategy for solving the reactive optimal power flow problem", Int. J. Electr. Power Energy Syst., vol. 66, p. 235-246, mar. 2015.

[36] I. Griva, "Numerical Experiments with an Interior-Exterior Point Method for Nonlinear Programming", Comput. Optim. Appl., vol. 29, n 2, p. 173 195, nov. 2004.

[37] A. Monticelli e W. H. E. Liu, "Adaptive movement penalty method for the Newton optimal power flow", IEEE Trans. Power Syst., vol. 7, n 1, p. 334-342, fev. 1992.

[38] A. Melman e R. Polyak, "The Newton modified barrier method for QP problems", Ann. Oper. Res., vol. 62, n 1, p. 465-519, dez. 1996.

[39] "Power Systems Test Case Archive - UWEE". [Online]. Disponível em: http://www2.ee.washington.edu/research/pstca/. [Acessado: 13-abr2017].

[40] J. Gilbert, C. Moler, e R. Schreiber, "Sparse Matrices in MATLAB: Design and Implementation", SIAM J. Matrix Anal. Appl., vol. 13, n 1, p. 333-356, jan. 1992.

[41] "MATLAB Documentation". [Online]. Disponível em: https://www.mathworks.com/help/\%20matlab/ref/mldivide.html. [Acessado: 13-abr-2017].

[42] A. Abusorrah, "The Application of the Linear Adaptive Genetic Algorithm to Optimal Power Flow Problem", Arab. J. Sci. Eng. Springer Sci. Bus. Media BV, vol. 39, nº 6, p. 4901-4909, jun. 2014.

[43] V. A. de Sousa, E. C. Baptista, e G. R. M. da Costa, "Loss minimization by the predictor-corrector modified barrier approach", Electr. Power Syst. Res., vol. 79, nº 5, p. 803-808, maio 2009.

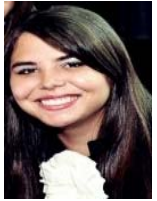

Jéssica Antonio Delgado, é graduada em Matemática pela Universidade do Sagrado Coração - USC, Bauru- SP, em 2013, tendo obtido o título de mestre em Engenharia Elétrica, em 2016, pela Universidade Estadual Paulista-UNESP, Bauru- SP. Atualmente é estudante de doutorado em Engenharia Elétrica da Universidade Estadual Paulista - UNESP, Bauru, onde desenvolve pesquisas na área de otimização, com métodos de barreira logarítmica e barreira logarítmica modificada, aplicados em sistemas de energia.

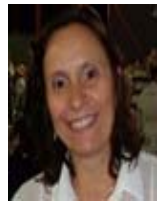

Edméa Cássia Baptista, é graduada em Matemática pela Universidade Estadual Paulista - UNESP - SP, em 1988, tendo obtido o título de mestre em Ciências da Computação, em 1993, e doutora em Engenharia Elétrica, em 2001, ambos pela Universidade de São Paulo - USP. Atualmente é Professora Adjunta do Departamento de Matemática da UNESP, em Bauru, onde desenvolve pesquisas nas áreas de otimização, programação não linear, Fluxo de Potência Ótimo e planicidade.

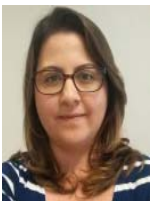

Gabriela Fernanda Bregadioli, é graduada em Matemática pela Universidade Estadual Paulista - UNESP, Bauru- SP, em 2012, tendo obtido o título de mestre em Engenharia Elétrica pela mesma universidade, em 2015. Atualmente, é estudante de doutorado pelo programa de Pós Graduação em Engenharia Elétrica da Universidade Estadual Paulista - UNESP, Bauru$\mathrm{SP}$, onde desenvolve pesquisas nas áreas de planejamento e otimização de sistemas de energia em mercados de energia.

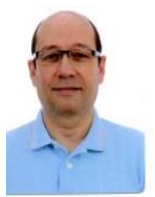

Leonardo Nepomuceno, é graduado em Engenharia Elétrica pela Universidade Federal de Uberlândia - UFU- MG, Brasil,em 1990, tendo obtido os títulos de mestre e doutor em Engenharia Elétrica pela Universidade Estadual de CampinasUNICAMP, Campinas - SP, Brasil, respectivamente em1993 e 1997. No período de 1997 a 2000, o professor atuou em projeto de pós-doutorado na UNICAMP. Atualmente, o professor desenvolve pesquisas na Universidade Estadual Paulista - UNESP0, Bauru SP, nas áreas de planejamento e otimização de sistemas de energia em mercados de energia.

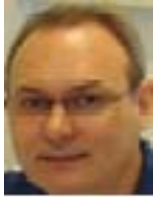

Antonio Roberto Balbo, é graduado em Matemática Universidade Estadual Paulista - UNESP - SP, em 1986, tendo obtido seu título de mestre em Ciência da Computação, em 1991 e seu título de doutor em Engenharia de Estruturas em 1998, ambos pela Universidade de São Paulo -USP. Atualmente é Professor Adjunto do Departamento de Matemática da UNESP, em Bauru, onde desenvolve pesquisas nas áreas de métodos primal-dual previsor-corretor de pontos interiores/exteriores aplicados em sistemas de energia.



Edilaine Martins Soler, é bacharel em Matemática pela Universidade de São Paulo - USP, em 2006, tendo obtido o título de mestre em Ciência da Computação e Matemática Computacional, em 2008, e o título de doutora em Engenharia Elétrica, em 2011, ambos pela Universidade de São Paulo USP. Atualmente é Professora Assistente da Universidade Estadual Paulista - UNESP - SP, onde desenvolve pesquisas nas áreas de otimização, atuando principalmente nos seguintes temas: programação não linear, variáveis discretas e problema de Fluxo de Potência Ótimo. 